\title{
Supporting Information: Statistical Inference of Transport Mechanisms and Long Time Scale Behavior from Time Series of Solute Trajectories in Nanostructured Membranes.
}

\author{
Benjamin J. Coscia ${ }^{1}$, Christopher P. Calderon ${ }^{1,2}$, and Michael R. Shirts ${ }^{1}$ \\ ${ }^{1}$ Department of Chemical and Biological Engineering, University of Colorado Boulder, \\ Boulder, CO 80309, USA \\ ${ }^{2}$ Ursa Analytics, Inc., Denver, CO 80212, USA
}

\section{Contents}

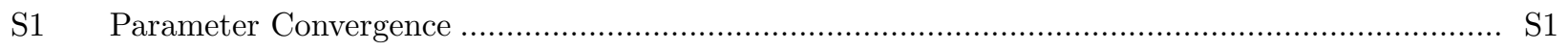

S2 Tips for Reliable State Sequence Identification …......................................................... S4

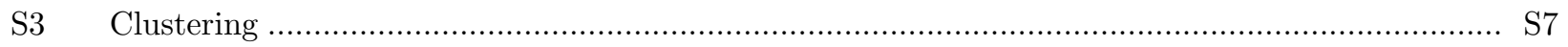

S3.1 Agglomerative Clustering Versus Gaussian Mixture Models................................................. S7

S3.2 Choosing Linkage Criteria and the Number of Clusters ..................................................... S8

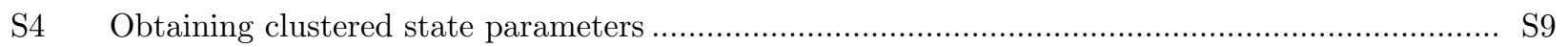

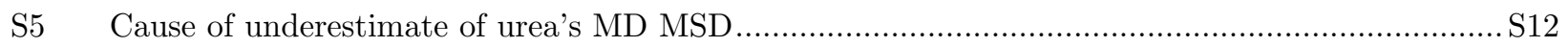

S6 Influence of number of clusters on qualitative hybrid trajectories ................................................ S12

S7 Deviations of the HDP-AR-HMM from Molecular Motion ............................................................ S13

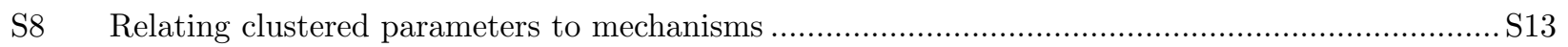

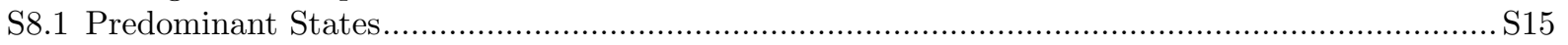

\section{S1 Parameter Convergence}

The state sequence appears to converge within 500-1000 IHMM iterations. To be certain, we ran 2000 iterations of the IHMM algorithm in order to arrive at a finalized state sequence. Stabilization of the VAR parameters indicates that the state sequence has converged. In Figure S1, we plot the diagonal entries of the $A$ and $\Sigma$ matrices as well as the entries of the mean vector $\mathbf{c}$, for three different states identified in a 3D methanol trajectory. States with a relatively small number of observations are more highly influenced by the boundaries of the state segments and generally lead to much higher variance of the $A$ and $\Sigma$ parameters. In these cases, the mean is a more reliable indicator of convergence since stabilized means imply that the fluctuations assigned to each state come from motion about a static location.

When we fix the state sequence during the steps of the procedure following the initial state sequence determination, the parameter estimates converge within only a few steps. In Figure S2, we plot the entries of $A$ and $\Sigma$ as a function of IHMM iteration. Some states are sampled far more frequently than others. High sampling frequently results in higher certainty converged parameter estimates. Due to fast convergence, we only ran 100 iterations of the inference behavior when the state sequence is fixed. 

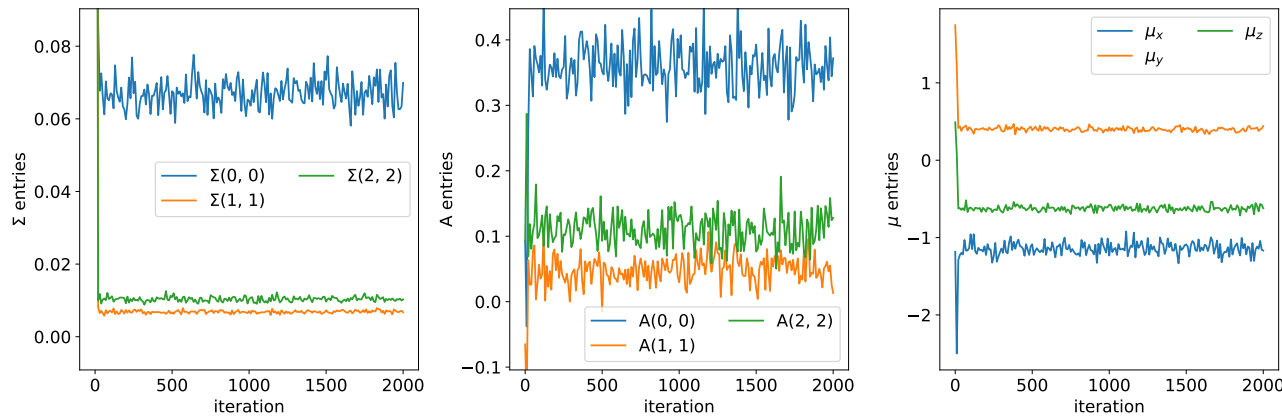

(a) 706 total emissions
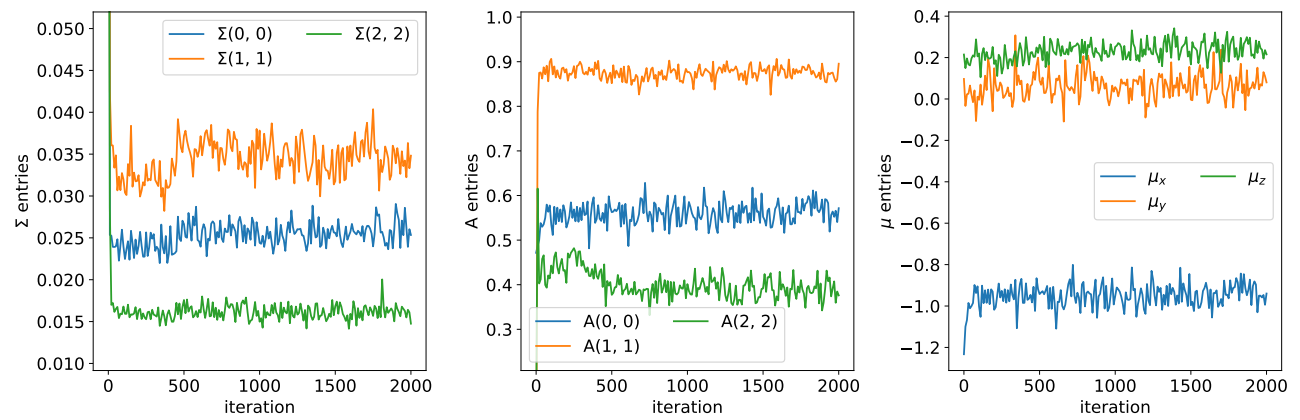

(b) 1000 total emissions
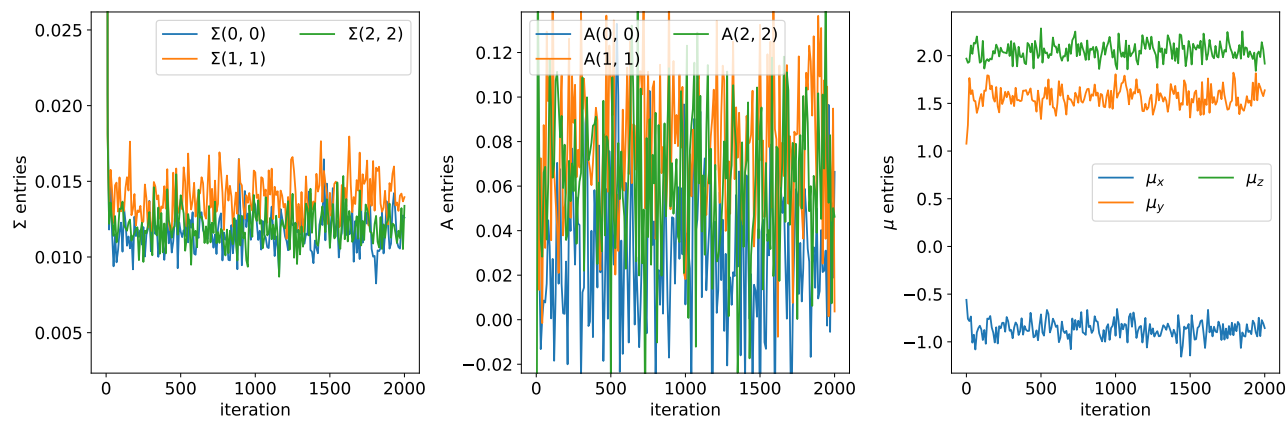

(c) 201 total emissions

Figure S1: Given a sufficient number of observations for a given mode, the VAR parameters appear to converge within $500-1000$ iterations. States with fewer observations are more highly influenced by the boundaries of the state segments which can lead to much higher fluctuations in the VAR parameters. 

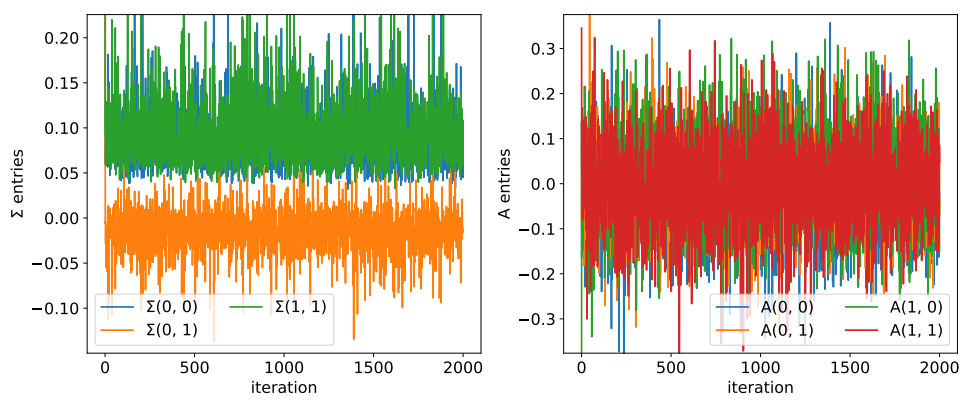

(a) 15 total emissions
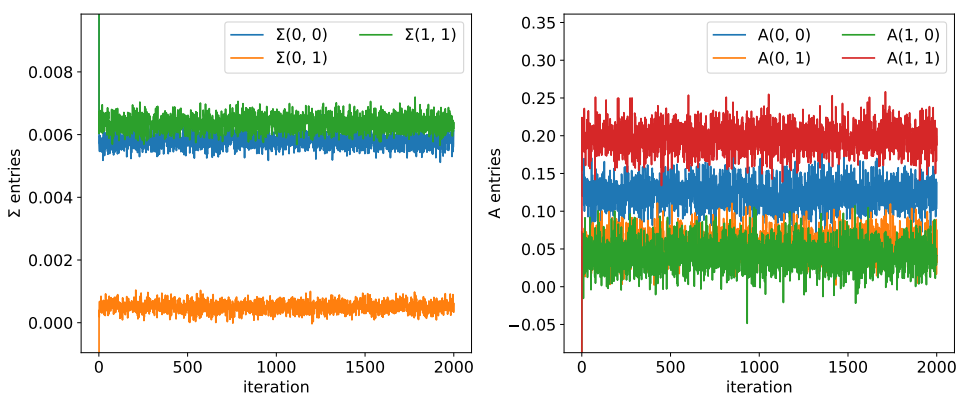

(b) 1289 total emissions
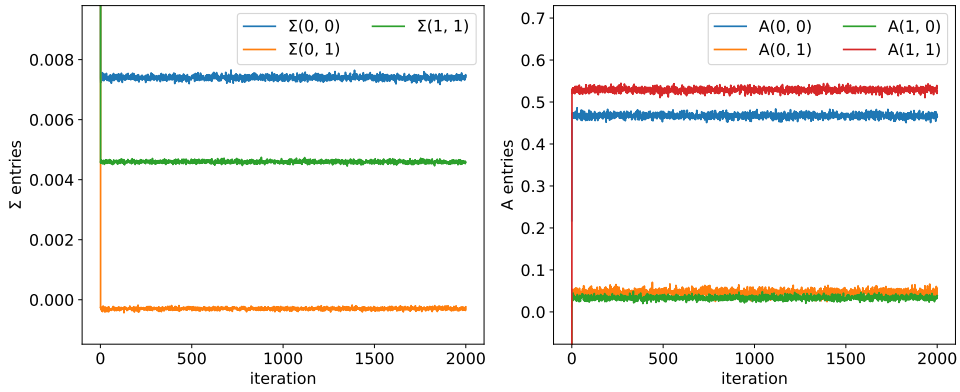

(c) 24468 total emissions

Figure S2: The VAR parameters converge quickly when we fix the state sequence. As the total number of emissions observed from each state increases, the uncertainty in the converged parameters estimates decreases. 


\section{S2 Tips for Reliable State Sequence Identification}

The IHMM is quite powerful on its own, but requires some critical thinking by the user in order to ensure maximum effectiveness. One can obtain the best results with some simple checks and, where necessary, improved state sequence initialization.

Choosing Prior Parameters: The states identified by the IHMM are heavily influenced by the Gaussian prior placed on $\mathbf{c}$ in Equation 1 of the main text. The entries of $A$ and $\Sigma$ do not vary over a wide range, so the final parameters were relatively insensitive to the priors. In order to maximally automate the IHMM procedure, we attempted to parameterize the prior on $\mathbf{c}$ in an intelligent way. The prior parameters should be chosen such that the mean level of each state lies within a region of reasonable probability of the prior (see Figure S3). In each dimension, we defined the prior mean to lie halfway between the maximum and minimum of each trajectory dimension. To parameterize the prior's variance in each dimension, we defined the maximum and minimum to be 2 standard deviations from the prior mean. Although this approach has worked quite well for the data in this work, it is important to check the results to determine whether further adjustments to the prior might be needed.

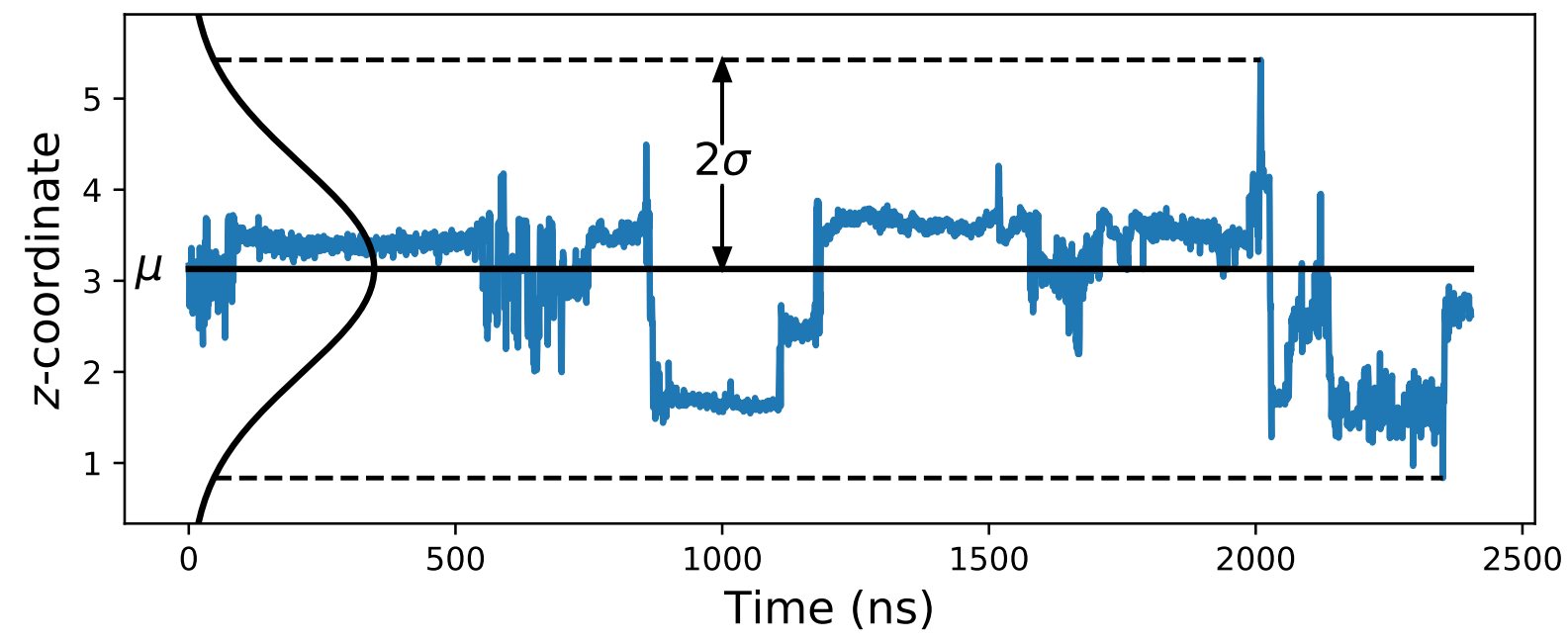

Figure S3: The parameters of the prior on the mean vector, $\mathbf{c}$, (black line), which represents the coordinates at which solute are trapped, should be chosen such that the mean levels of each state identified in the trajectory (blue line) lie within regions of the prior with reasonable probability. We chose the mean of the prior as halfway between the maximum and minimum (shown by the dashed lines) of each trajectory dimension. We chose $\sigma$ of the prior by defining the maximum and minimum to be 2 standard deviations from the mean.

Seeding the initial state sequence: We always initialize the prior on $\mathbf{c}$ as described above but in some particularly tricky cases, the initial state sequence estimate is so poor that qualitative trajectory realizations and MSD predictions are irreconcilable with MD. A simple way to identify bad parameterizations is to check whether the MD MSD of individual trajectories can be reasonably reproduced by realizations of the HDPAR-HMM fit to the same trajectory. In Figure S4a, the MSD predicted by the HDP-AR-HMM dwarfs that calculated from MD, due to rapid switching between states in the final state sequence.

We can improve the final state sequence estimate by seeding the initial state sequence with a guess made by applying the HDP-AR-HMM to smaller segments of the trajectory. In Figure S5, we illustrate this process. In Figure S4c, the MSD predicted by the HDP-AR-HMM is much more consistent with MD after applying this approach. 


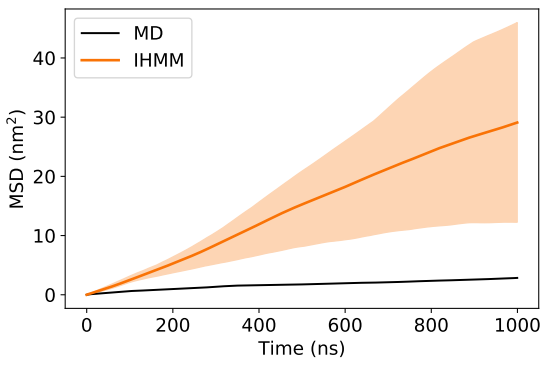

(a)

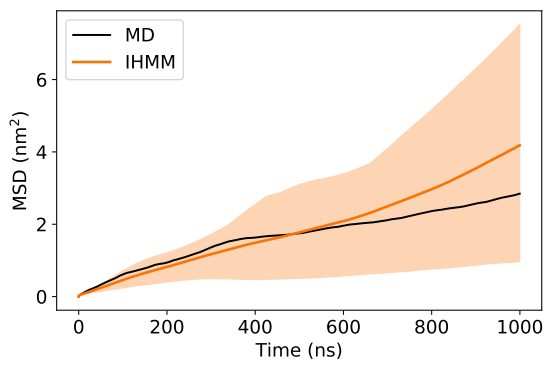

(c)

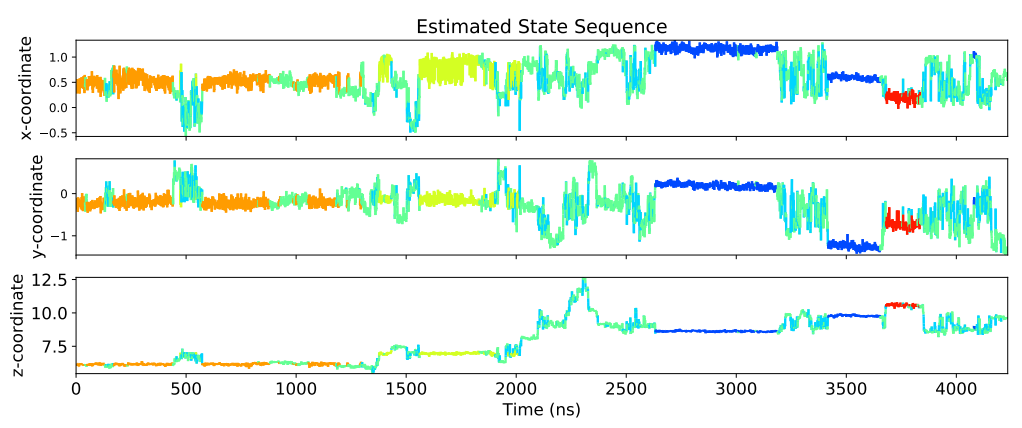

(b)

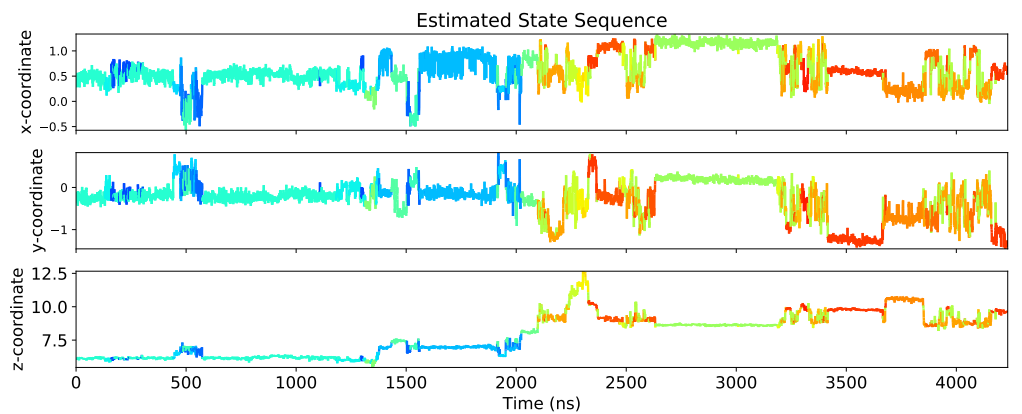

(d)

Figure S4: $(\mathrm{a}, \mathrm{b})$ In some cases, the state segmentation leads to over- or under-prediction of the MSD, usually due to poor initialization of the state sequence. In (b), state transitions are too frequent, particularly between the light blue and light green states, and ultimately causes an over-prediction of the MD MSD. (c) We can greatly improve MSD predictions by giving the HDP-AR-HMM a good guess at the initial state sequence. (d) A better initial state segmentation via seeding gives more reasonable estimates of the final state sequence as illustrated in Figure S5. 


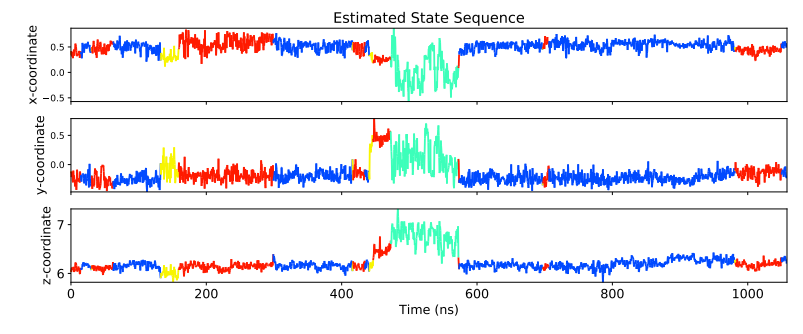

(a) Found 4 Unique States

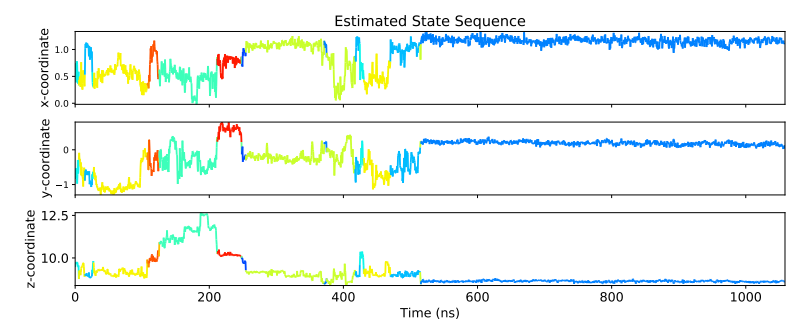

(c) Found 13 Unique States

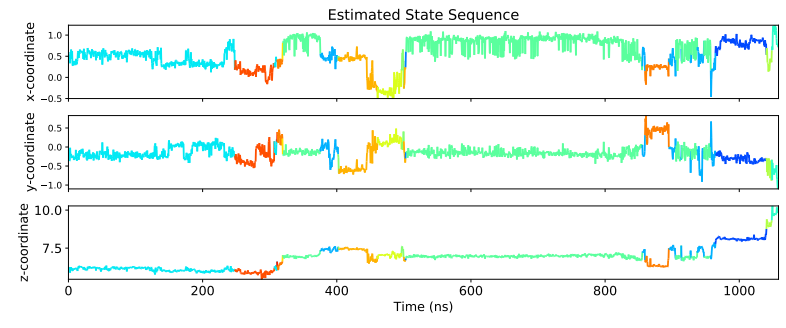

(b) Found 14 Unique States

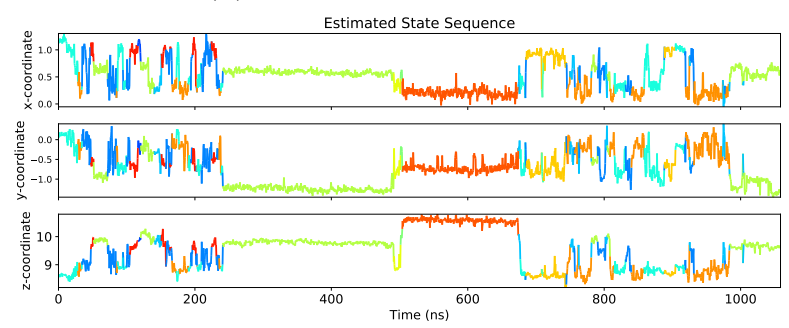

(d) Found 12 Unique States
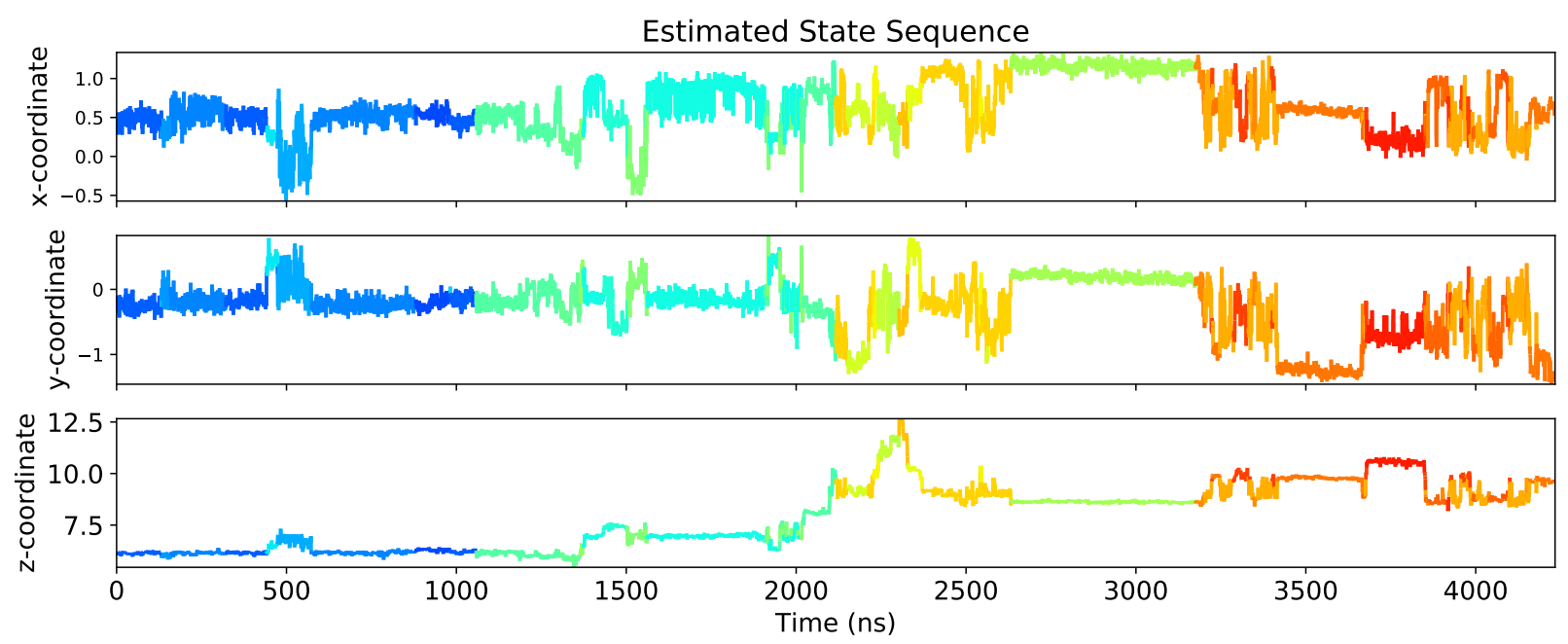

(e) Final state sequence seeded with states found in (a)-(d)

Figure S5: In (a-d), we show the state sequences which result from 5 iterations of the HDP-AR-HMM on each of the four quarters of the center-of-mass trajectory in (e). We use the concatenation of the four state sequences as the initial sequence for the HDP-AR-HMM inference run on the fully trajectory in (e). 


\section{S3 Clustering}

\section{S3.1 Agglomerative Clustering Versus Gaussian Mixture Models}
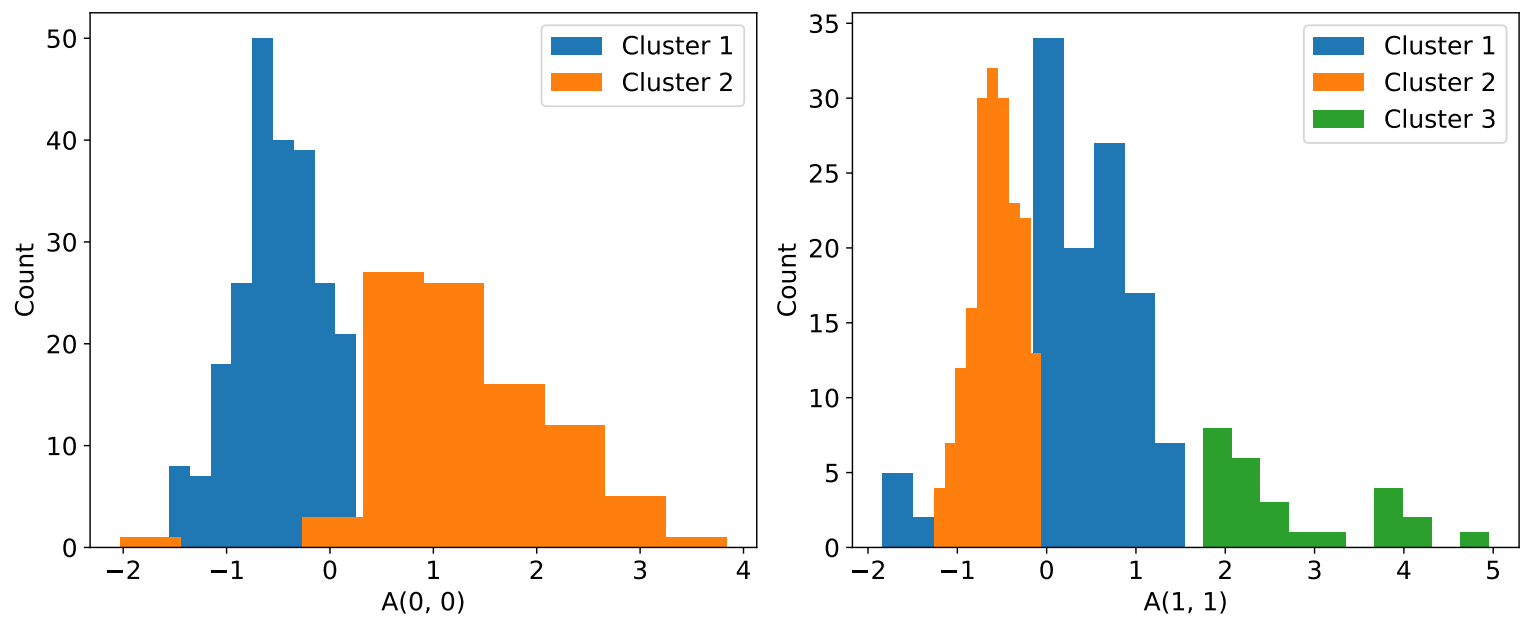

(a) Dirichlet process Gaussian mixture model
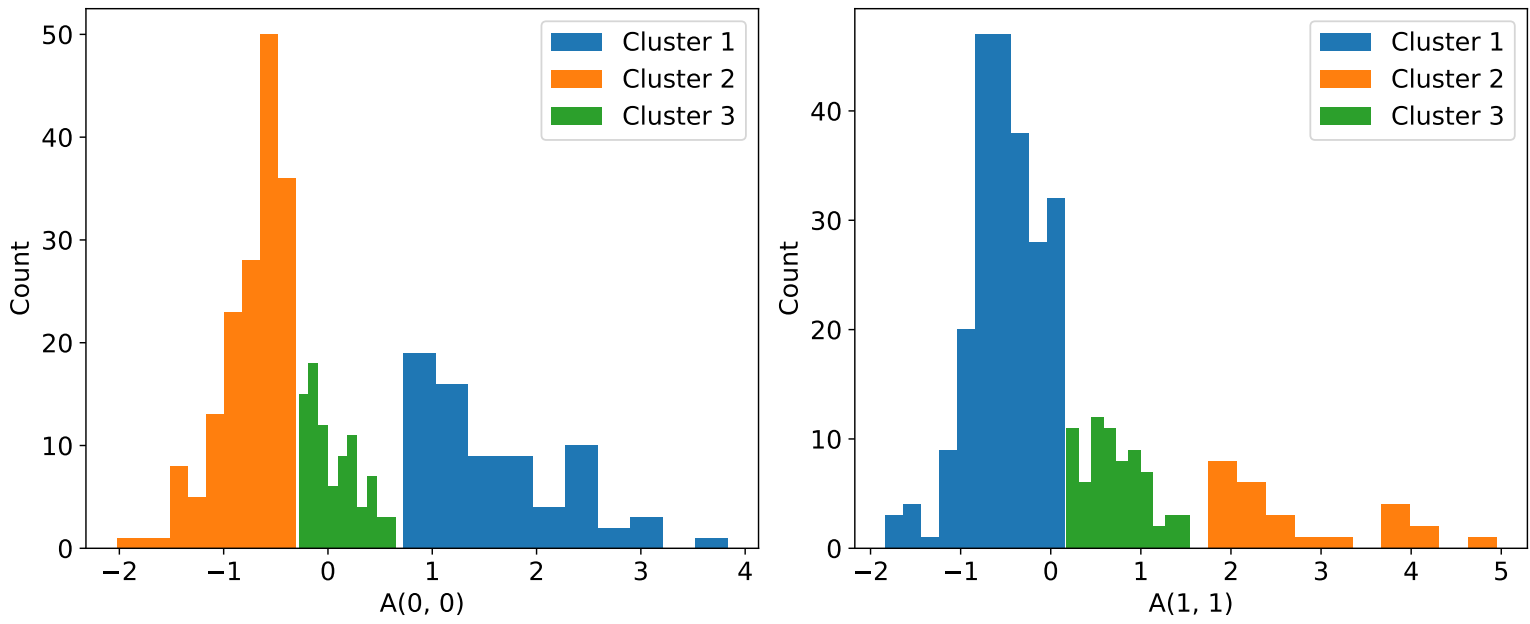

(b) Agglomerative Clustering

Figure S6: To reduce the state space, we prefer agglomerative clustering over non-parametric Dirichlet process Gaussian mixture modeling. In the plots above we show the results of clustering the diagonal entries of the autoregressive coefficient matrices, A, of methanol. (a) Dirichlet process Gaussian mixture models tend to delocalize the clusters in parameter space since the Gaussians can overlap. Agglomerative clustering prevents overlap of clusters and ensures that all states within each cluster have similar parameters. 

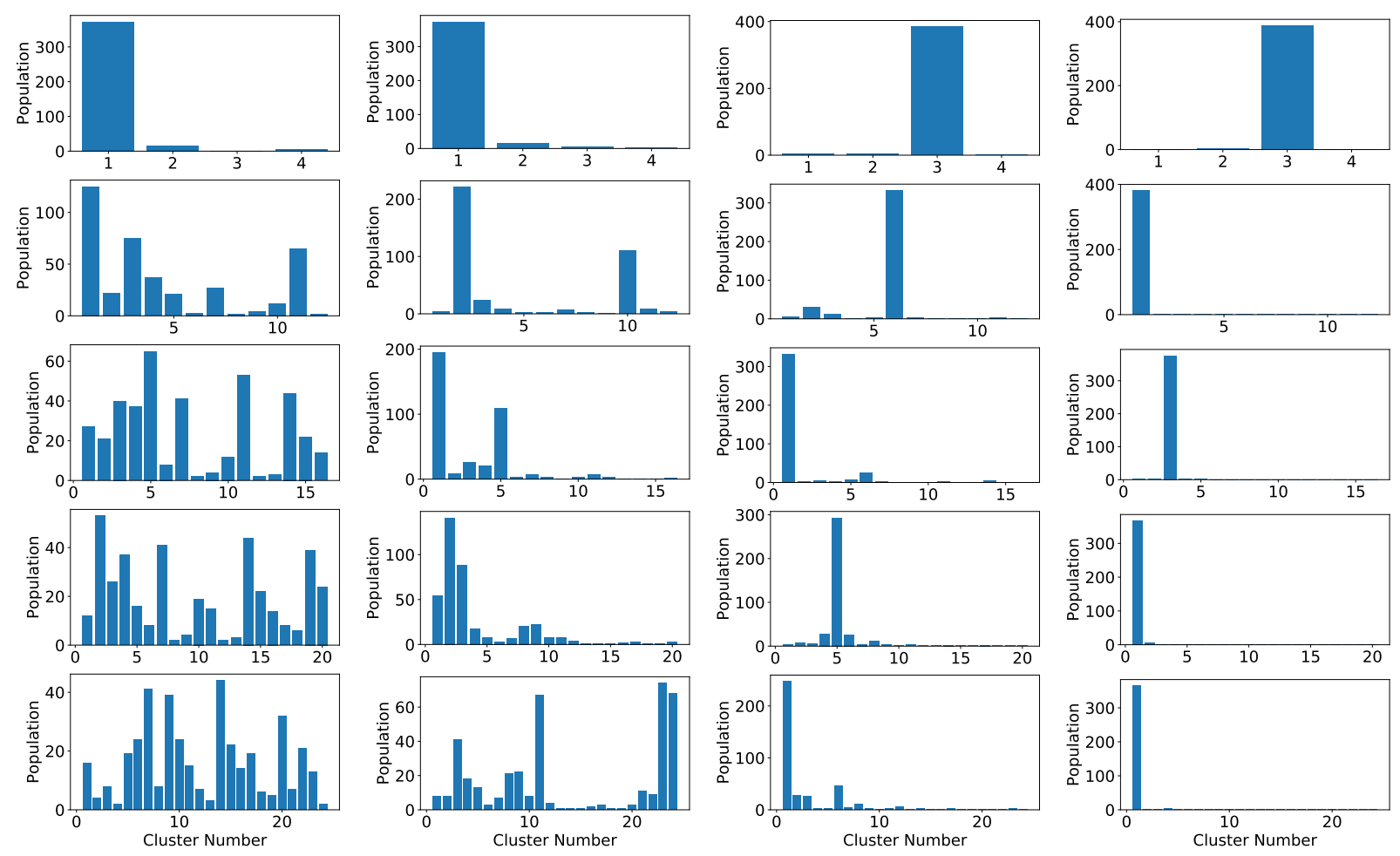

(a) 'ward'

(b) 'complete'

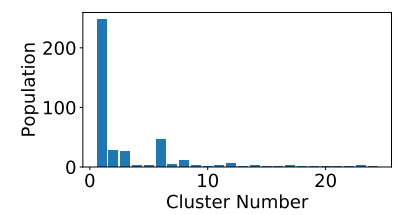

(c) 'average'

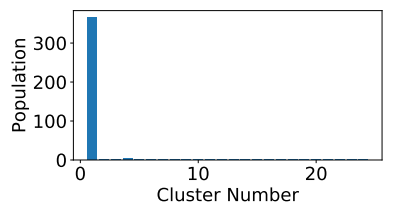

(d) 'single'

Figure S7: In each column we tested a different linkage criteria for clustering of the methanol parameters. In each row, we varied the number of total clusters. Both 'ward' and 'complete' linkage appear to induce clustering while the 'average' and 'single' criteria tend to concentrate parameters in only a fraction of the available clusters.

\section{S3.2 Choosing Linkage Criteria and the Number of Clusters}

In this section, we determine the optimal linkage criteria and number of clusters for studying methanol's parameters. We applied the same workflow in order to make analogous decisions for ethylene glycol, urea and acetic acid. In all cases, we found that the 'ward' linkage criteria resulted in the most useful clustering. We chose to use 10 clusters for each solute, the minimum number of clusters, by our analysis, needed to adequately distinguish state dynamics.

In order to cluster the parameter sets, we need to determine the linkage criteria that will be used to measure the distance between clusters as well as an appropriate total number of clusters. In Figure S7, we show the result of clustering with each type of linkage criteria available with the

sklearn.cluster. AgglomerativeClustering class of the scikit-learn python package. It is clear that both the 'average' and 'single' linkage criteria do not sufficiently cluster the data. The 'ward' criteria appears to result in the highest number of large population clusters.

We can help to further narrow our decision of linkage criteria by analyzing the parameters of the clusters which it produces. In particular, we should look for well-distinguished clusters on the radial means so that it will be easier to connect solute behavior to the membrane pore structure. One way to concisely visualize the radial clusters is by their spread. An optimal number of clusters should have the same spread in radial means as the unclustered data. In Figure S8a, we show that 'ward' linkage results in the highest spread in the radial means of the clusters across a range of 4-30 total clusters. For a low to intermediate number of clusters, the spread of 'ward' clusters is significantly larger than of the other linkage methods. Therefore, we have chosen to use 'ward' clustering.

We use the silhouette score in order to aid us in choosing the total number of clusters. In Figure S8b, we plot the silhouette score as a function of the total number of clusters using the 'ward' linkage criteria. 


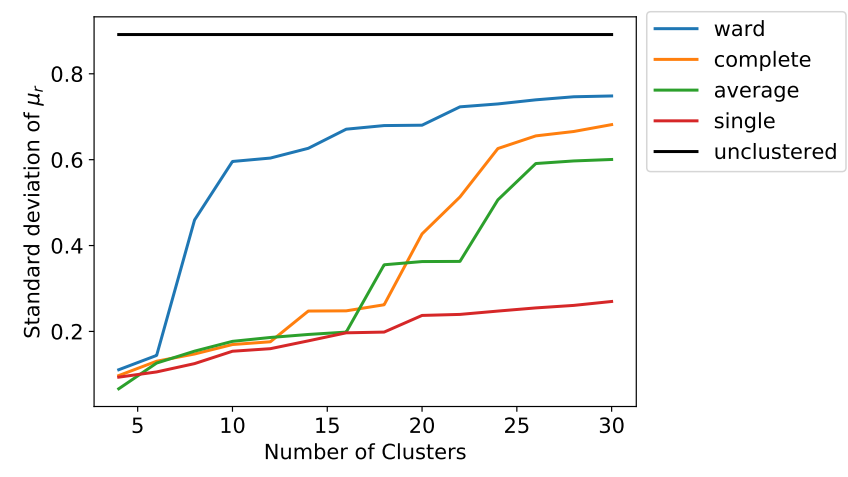

(a)

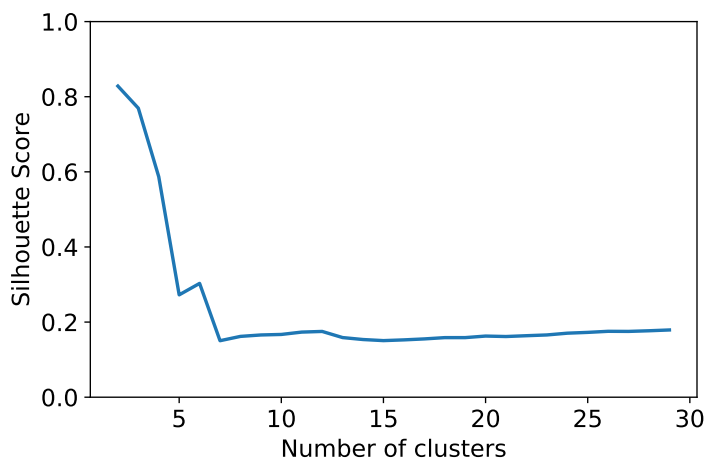

(b)

Figure S8: (a) The 'ward' linkage criteria maintains the highest standard deviation in radial means, $\mu_{r}$, of the clusters for a given total number of clusters as compared to other linkage criteria. (b) The silhouette score, used to evaluate the quality of clustering with the 'ward' distance criteria drops precipitously as a function of the chosen total number of clusters before plateauing.

A wilhouette score of 1 indicates the best clustering possible, values near 0 indicate overlapping clusters, and negative values indicate that samples belong to the wrong clusters. For the methanol parameters, the silhouette score drops off quickly with number of clusters before plateauing around 7 total clusters. However, given the low spread in $\mu_{r}$, it is not clear that using 7 or less clusters will give useful clustering results.

We incorporate qualitative feedback into the decision on the number of clusters. Despite its high silhouette score, Figure S9a illustrates that using 4 clusters clearly does not distinguish dynamical modes. We do not see visually acceptable clustering on the example trajectory until we use at least twenty total clusters (Figure S9b).

\section{S4 Obtaining clustered state parameters}

Parameterization the clustered states with the HDP-AR-HMM is a multi-stepped procedure. In the steps and figures that follow, we graphically illustrate the procedure which is described with additional detail in Section 2.3 of the main text.

1. Parameterize in $x, y, z$ coordinates with $x, y$ coordinates relative to the nearest pore center (see Figure S10a).

2. Cluster the VAR parameters from the states found in all 24 trajectories (See Section 2.3 of the main text). Reassign the state sequence so that segments which belong to the same cluster are labeled the same across all solute trajectories.

3. Zero the trajectories. First, zero out the $y$ dimension by rotating each segment of the trajectory, as partitioned before clustering, about the $z$ axis, in order to align the mean $x y$ vector with the $x$ axis (see Figure S10b). Then, subtract the mean in $x$ and $z$ (Figure S10c).

4. Fix clustered state sequence and infer parameters of each state, assuming a mean of zero for all states (see Figure S10d). 

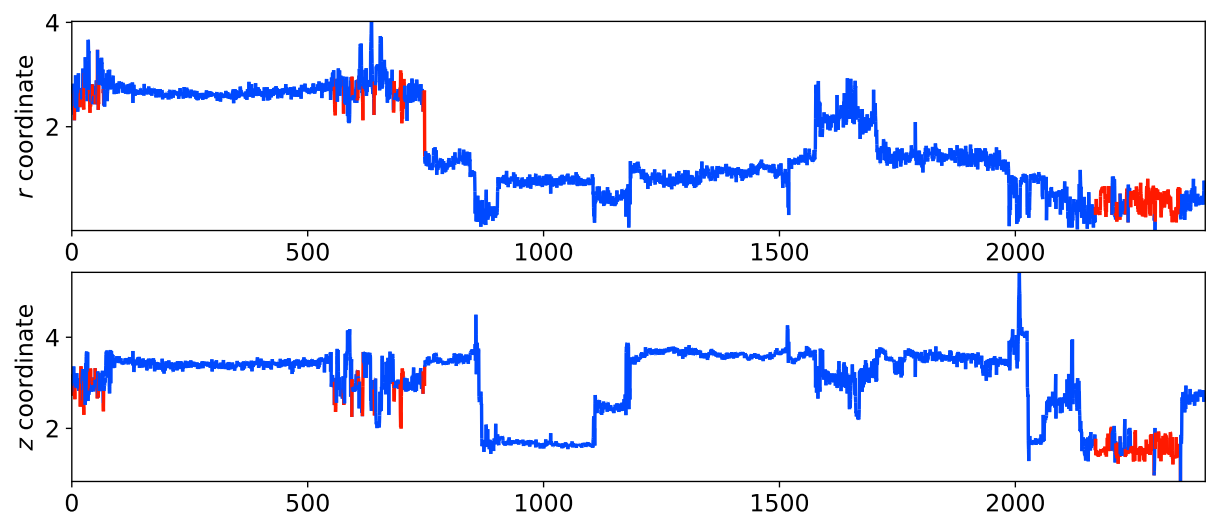

(a) 4 total clusters
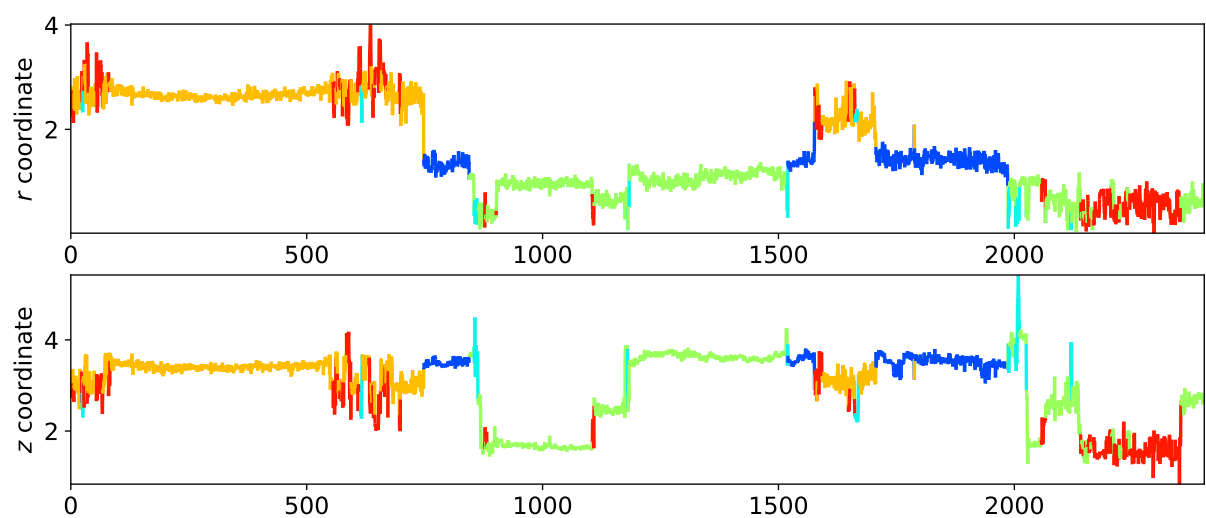

(b) 10 total clusters

Figure S9: Although the silhouette score for four total clusters is high, we do not see adequate distinction between clusters until we group the parameters from the 24 trajectories into at least 10 total clusters. 


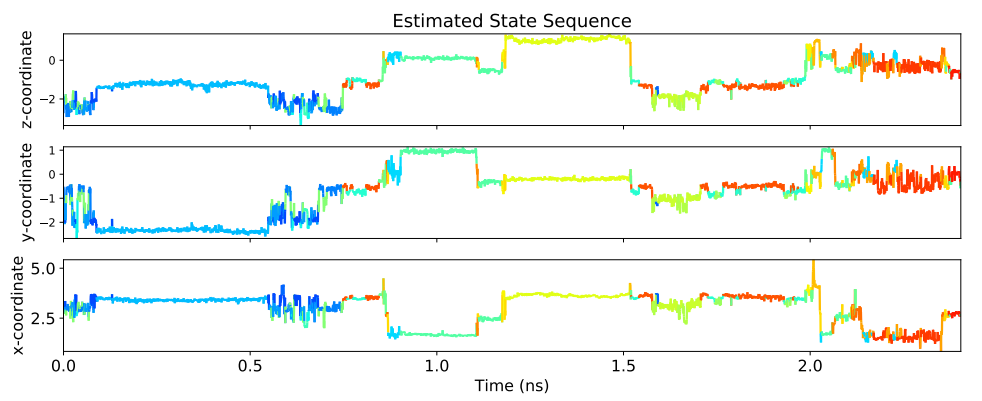

(a)
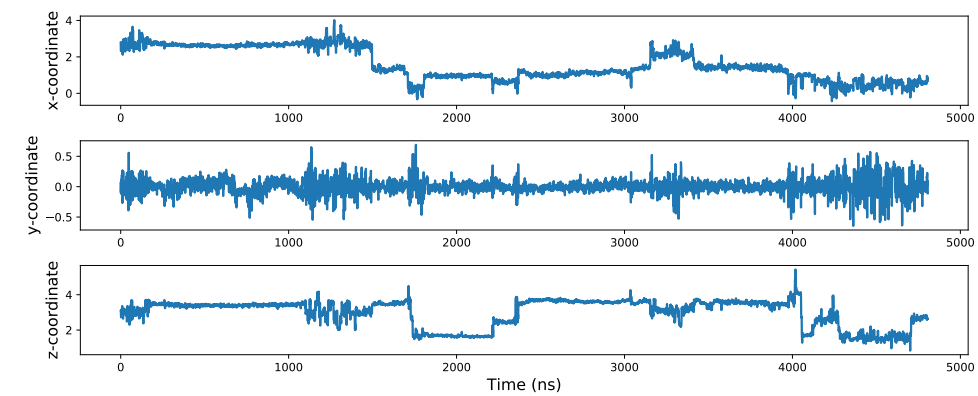

(b)
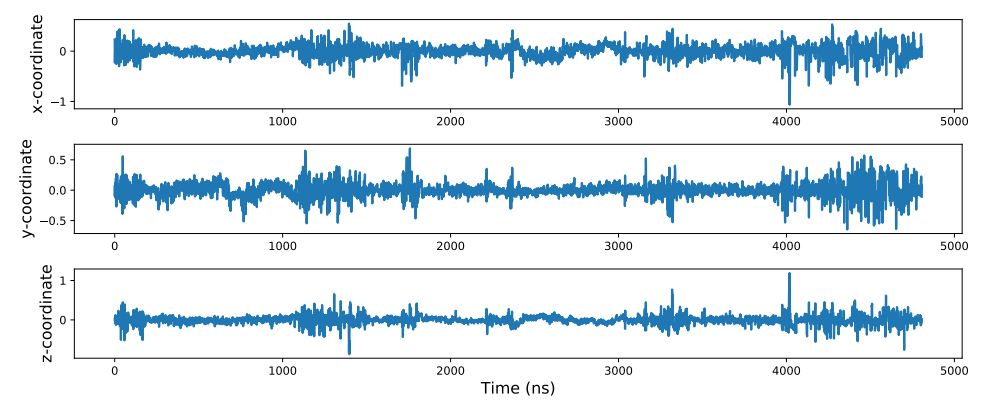

(c)

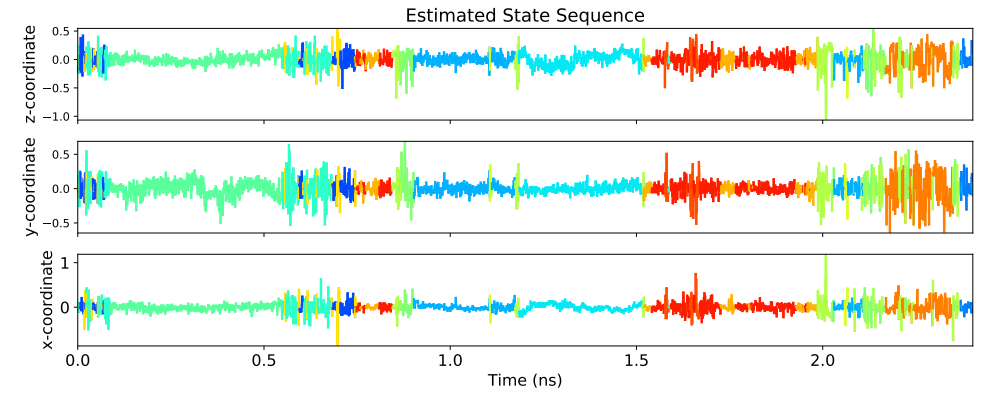

(d)

Figure S10: In the above plots, distinct colors correspond to distinct state behavior. (a) First we parameterize the trajectories in terms of the $x, y, z$ solute center-of-mass coordinates with the $x$ and $y$ coordinates relative to the nearest pore center. The parameters of these states are used to predict the unclustered MSDs according to method 1 described in Section 2.4 of the main text. (b) Next, we zeroed out the $y$ dimension by rotating each segment of the trajectory, as partitioned before clustering, about the $z$ axis, in order to align the mean $x y$ vector with the $x$ axis. (c) We then subtracted the $x$ and $y$ means of each segment in order to fully zero the trajectory. (d) Finally, we applied the inference component of the HDP-AR-HMM in order to infer the parameters of the clustered states. 


\section{S5 Cause of underestimate of urea's MD MSD}

Realizations of the HDP-AR-HMM underestimate the MD MSD of urea due to MD trajectories with axial motion correlation that is likely uncharacteristic and unable to be captured by ensembles of stochastic HDPAR-HMM realizations. One such trajectory is plotted in Figure S11. Axial hops appear positively correlated, jumping consistently in the negative $z$ direction, leading to a high MSD. The parabolic trajectory of the MD MSD curve implies underlying super-diffusion which would require some kind of facilitating driving force. It is likely that running the trajectory longer would reduce the slope of the MSD curve, making it more consistent with the HDP-AR-HMM prediction.

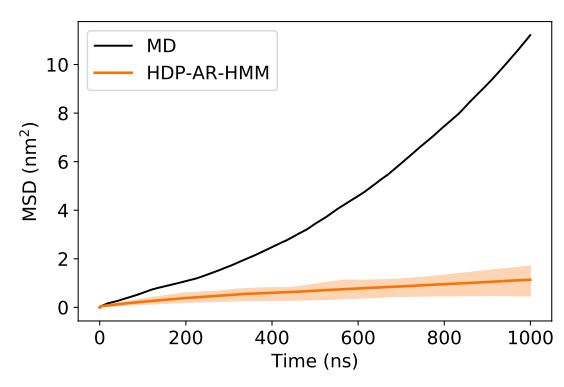

(a)

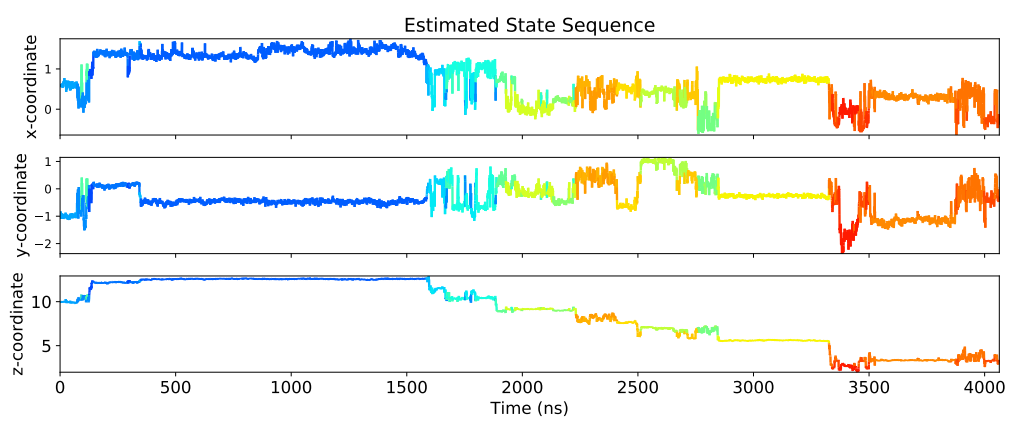

(b)

Figure S11: (a) The HDP-AR-HMM under-predicts the MD MSD of urea when fit to the trajectory in (b) because axial hops are highly correlated, behavior our model is unable to reproduce.

\section{S6 Influence of number of clusters on qualitative hybrid trajecto- ries}

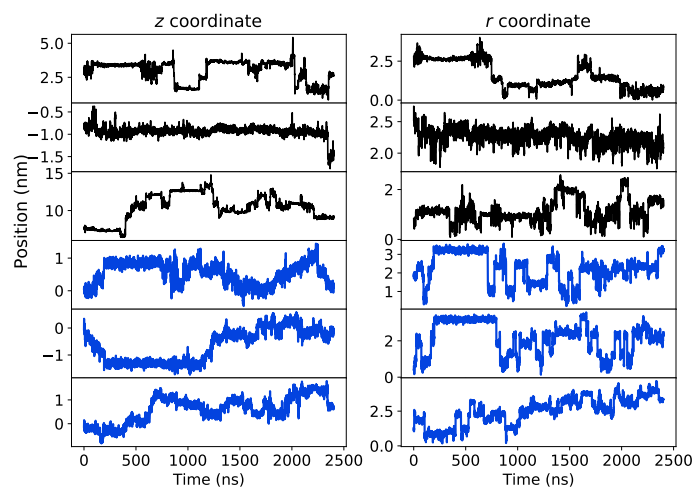

(a) 20 clusters
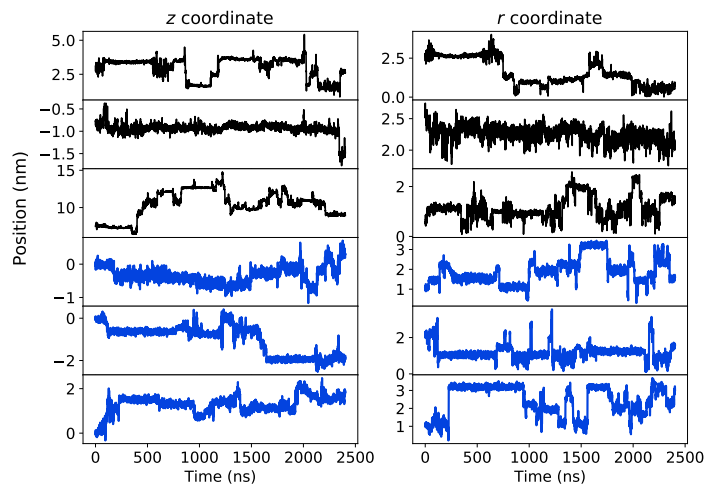

(b) 30 clusters

Figure S12: The quality of hybrid trajectories resulting from clustered parameters sets qualitatively improves as the number of clusters is increased. This comes at the cost of a larger state space to interpret. 


\section{S7 Deviations of the HDP-AR-HMM from Molecular Motion}

At extremely short time lags, the MSD is generally over-predicted by the HDP-AR-HMM (see Figure S13a) because the VAR(1) model assumes multivariate Gaussian noise while the MD data suggests the noise is better modeled by more general Lévy stable noise. The first time lag of the MSD is equivalent to the variance of all observed fluctuations. The HDP-AR-HMM simulation appears to over-estimate the width of the hop distribution because the actual distribution of solute fluctuations has heavy tails with a smaller width (Figure S13b). The total distribution of hops is expected to be heavy tailed because it is a combination of hop distributions from many states with different variances. More interestingly, in Figure S13c, we show that the emission distribution of a single state picked out by the HDP-AR-HMM is fit better by a more general Lévy distribution while the emissions from HDP-AR-HMM realizations are Gaussian, as expected. An improvement to the HDP-AR-HMM that may allow dynamics more faithful to MD would use an autoregressive model with Lévy stable noise. However, this would be less computationally feasible due to lack of conjugate priors.

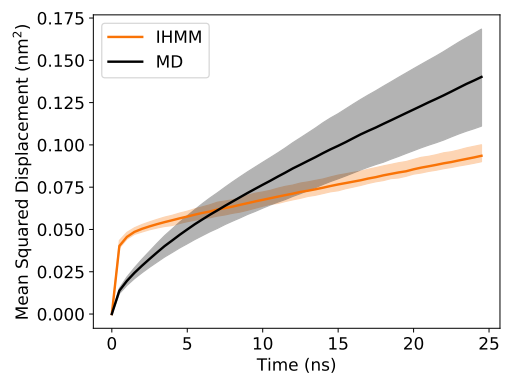

(a)

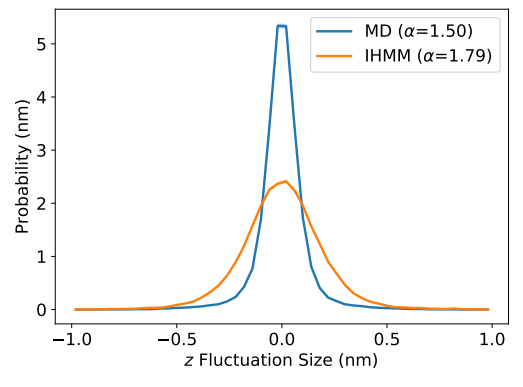

(b)

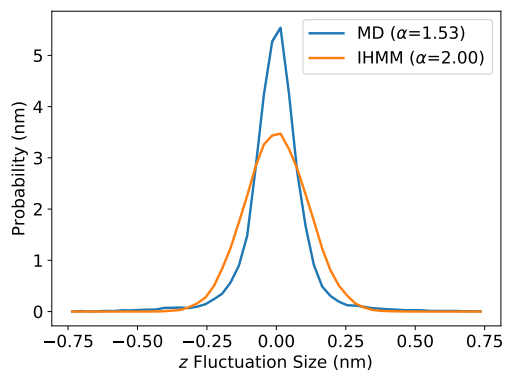

(c)

Figure S13: (a) At short time lags, the MD MSD (black) and the HDP-AR-HMM MSD (orange) differ significantly in their shape and curvature. (b) While the fluctuations in $z$ of both distributions are heavytailed, fitting well to zero-centered symmetric Lévy stable distributions with stability parameters, $\alpha$, the HDP-AR-HMM has a wider variance. (c) The distribution of fluctuations in a chosen state frequently visited by methanol is heavy tailed, but the HDP-AR-HMM is constrained to produce fluctuations from a multivariate normal distribution ( $\alpha=2$ in each dimension).

Also apparent from Figure S13a is that the curvature of the MD MSD is far more gradual than that generated by the HDP-AR-HMM. This is because the VAR(1) model is only correlated to its previous fluctuation while the MD simulations suggest that correlation persists for at least tens of nanoseconds. One may be able to reproduce the curvature at short time lags with higher order VAR models, but it becomes a much higher dimensional parameterization that would require significantly more data in order to produce stable results. Since we are interested in reproducing longer time scale behavior, it is not an issue of great concern.

\section{S8 Relating clustered parameters to mechanisms}

We can begin to hypothesize mechanisms by studying the behaviors defined by the state parameters. In Figure S14a, we plot representative dynamics of each of the five most visited states (see Figure 7 of the main text). The states exhibit a range of autoregressive and trapping behavior throughout the membrane pores.

Since the radial means are weighted averages of the states used to parameterize each cluster, for interpretive purposes, it is important to recognize that the means only provide clues towards the general location of the solute. For example, the radial mean of state 3 is $1.4 \mathrm{~nm}$ from the pore center. However, inspection of Figure 5 of the main text suggests that this type of behavior actually occurs radially throughout the pore. There are instances of state 3 less than $1 \mathrm{~nm}$ from the pore center and more than $2 \mathrm{~nm}$ from the pore center. In this case, the radial mean only suggests that this type of behavior happens in both regions somewhat equally. 

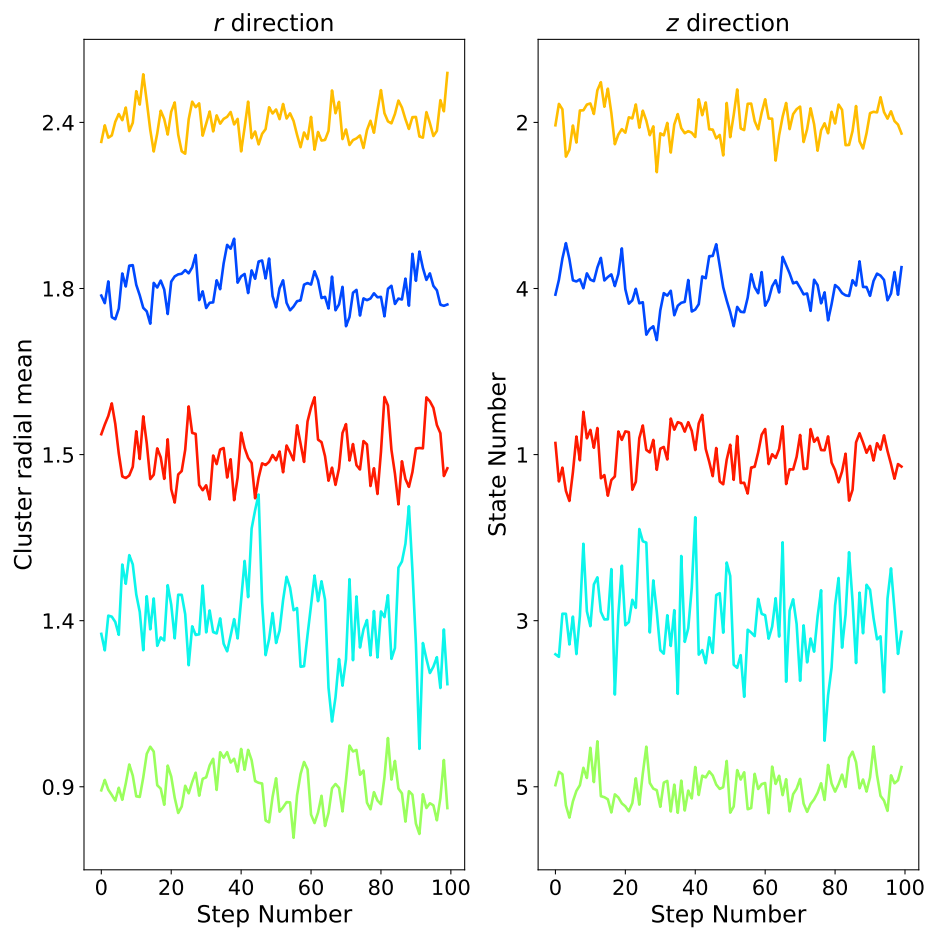

(a)

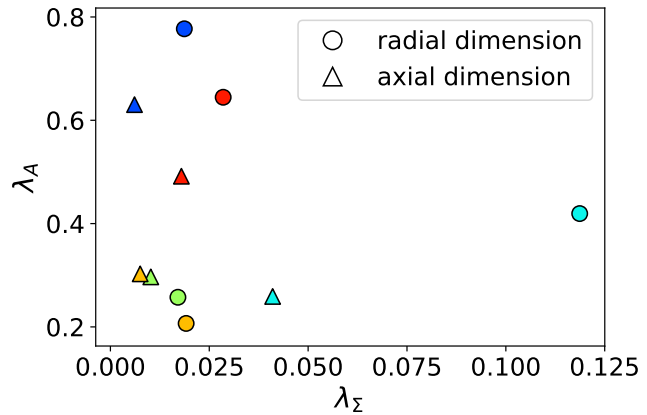

(b)

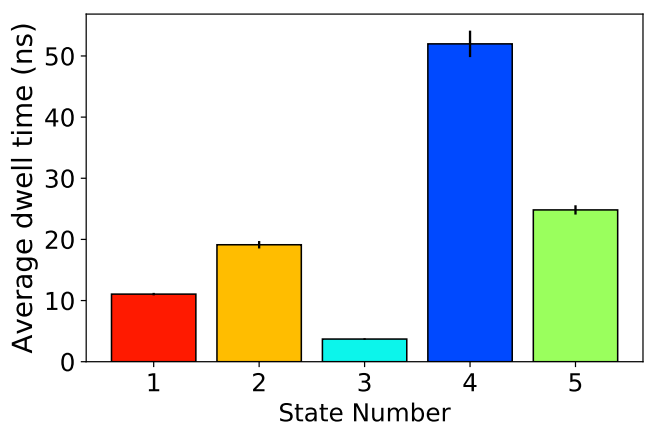

(c)

Figure S14: The 5 most visited states by methanol show a range of dynamical behavior. In (a), we show representative dynamics of these states. All time series have a mean of zero and are shifted to put the mean in line with the $y$-axis labels for the purpose of visualizing them. The $y$-axis labels of the $r$ coordinate plot specify the radial mean of each clustered state. All of the states pictured appear in the trajectory shown in Figure 5 of the main text and are colored to match. (b) We can begin to understand the solute behaviors demonstrated in (a) by understanding their VAR parameters. Higher values of $\lambda_{\Sigma}$ generally are indicative of large fluctuations and higher values of $\lambda_{A}$ indicate strong previous-hop dependence of the fluctuations. (c) Using Equation 2 of the main text, we estimated the expected time spent within each of the states.

Solutes exhibit a range of autoregressive behavior as shown by the representative solute trajectories in Figure S14a and the scatter plot in Figure S14b. In most cases, the eigenvalues of $A$ and $\Sigma$ appear nearly paired in their radial and axial dimensions, which implies nearly symmetric behavior with off-diagonals both matrices near zero. The most notable exception is state 3 , which has a much higher radial eigenvalue of the covariance matrix. Both eigenvalues of state 3's covariance are actually quite large. This implies relatively large fluctuations in the $z$ direction and even bigger fluctuations in the $r$ direction. All states have slightly lower covariance in the axial direction. It's likely easier for solutes to move laterally rather than to move up through the alkane chains. States 3 and 4 show a strong previous-hop dependence (high $A$ ) while the rest of the states have a somewhat weaker dependence.

Solutes stay trapped for various lengths of time. On average, state 4 has the longest dwell times and state 3 has the shortest dwell times while the rest have intermediate dwell times. This is somewhat supported by inspection of Figure 5, however, the expected dwell times of the clusters incorporate all 24 trajectories.

The local number density of heavy atoms surrounding solutes help round out methanol's mechanistic picture. Although hydrogen bonding interactions are relatively frequent, methanol molecules are unbound by any measurable electrostatic interactions about half or more of the simulation time. Figure S15 helps to illustrate how the local density of membrane components can aid in solute entrapment. When solutes experience long trapping times, the local number density of heavy atoms is larger. Even when hydrogen bonds break, surrounding alkane density likely helps keep the solutes in place allowing them to eventually reform. This can happen deep in the tails or close to the monomer head groups. Methanol molecules which 
experience State 3 have by far the smallest local number density which is likely responsible for their mobility, as suggested by the state's large covariance.

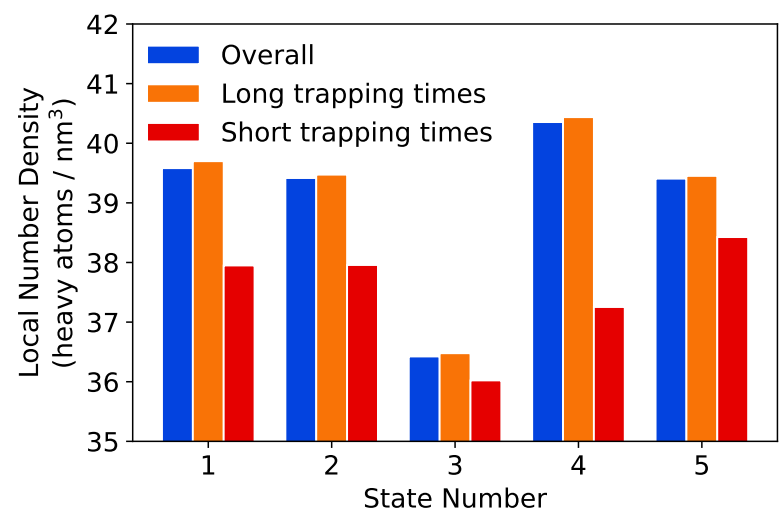

Figure S15: We measured the local number density of heavy atoms surrounding solutes in each state. In blue, we plot the local number density experienced by solutes averaged over all frames in which the solute occupied the given state. We broke down this overall average number density into number densities measured from instances when the state is occupied for long (orange) versus short (red) dwell time states. We define define 'long' dwells times as the 50th percentile of all dwell times in the given state, and short as all others. It is clear that solutes which experience longer dwells times are also surrounded by more heavy atoms on average.

\section{S8.1 Predominant States}

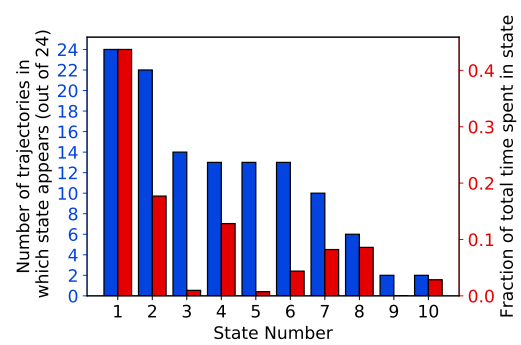

(a) ethylene glycol

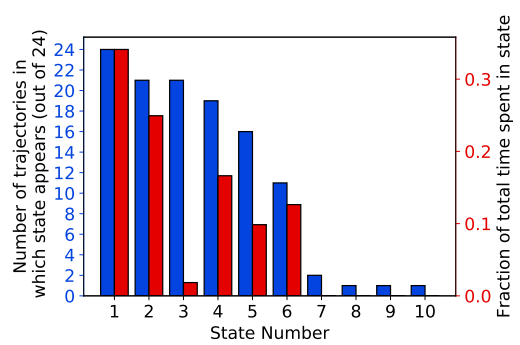

(b) urea

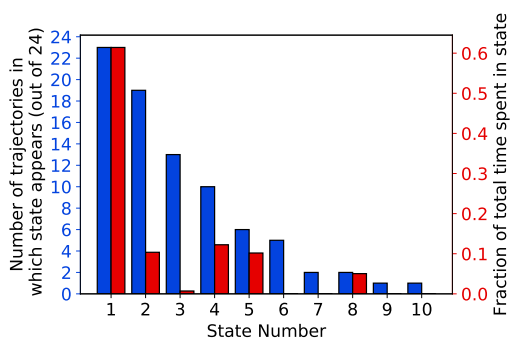

(c) acetic acid

Figure S16: MD trajectories sample the clustered states to varying degrees and for varied amounts of time. For each solute in (a), (b) and (c), we plot the total number of trajectories that sample a given state (blue) as well as the fraction of the total simulation time spent in that state. 